\title{
Estudo nutricional e socioeconômico de crianças em unidades de educação infantil
}

\author{
Michele Salles da Silva1', Débora Aparecida da Silva Santos², Jacqueline Pimenta Navarro³, \\ Gênesis Vivianne Soares Ferreira Cruz ${ }^{4}$, Dangllie Katherine Verdi ${ }^{5}$, Amanda de Oliveira Delfino ${ }^{6}$
}

\section{RESUMO}

Objetivo: realizar avaliação nutricional de crianças de 01 a 04 anos matriculadas em Unidades Municipais de Educação Infantil (UMEI) de Rondonópolis/MT e descrever a relação do perfil socioeconômico da família com o estado nutricional da criança. Método: estudo exploratório, descritivo com abordagem quantitativa. Foi utilizado critério de abordagem amostral de $30 \%$ de todas as crianças matriculadas no ano de 2013, com a seleção dos sujeitos por meio de sorteio de dois dos cinco polos existentes na região, totalizando 185 crianças. Resultados: foram avaliados os parâmetros de peso, altura e IMC (Índice de Massa Corporal), apresentando que grande maioria das crianças com IMC adequado, seguida de taxas relevantes de risco de sobrepeso, sobrepeso e magreza. Conclusão: 0 estudo demonstrou que apesar da predominância de 1 a 2 salários mínimos para o sustento das famílias pesquisadas, os índices nutricionais apresentaram-se adequados para a maioria das crianças que frequentam as UMEls.

Descritores: Criança, Avaliação Nutricional; Creches.

\section{Nutrition and children in socioeconomic study early childhood education units}

\begin{abstract}
Objective: To carry out nutritional assessment of children ranging from 01 to 04 years old enrolled in Early Childhood Municipal Units (UMEI) from Rondonópolis, Mato Grosso State, and to describe the family's socioeconomic profile relationship with the child's nutritional status. Method: an exploratory and descriptive study was conducted with a quantitative approach. Sampling approach was used in $30 \%$ of all children enrolled in 2013, with the selection of subjects from two of the five existing centers in the region, totaling 185 children. Results: we evaluated the weight, height and BMI (Body Mass Index) parameters, showing that most children with adequate BMI, followed by relevant rates of overweight risk, overweight and thinness. Conclusion: The study showed that despite 1 to 2 minimum wages was the predominant salary range of the surveyed families, nutritional indices were suitable for most children attending UMEls.
\end{abstract}

Descriptors: Children; Nutritional Assessment; Nursery School.

\footnotetext{
${ }^{1}$ Mestre em Saúde Coletiva pela Universidade Federal de Mato Grosso (UFMT), Cuiabá, Mato Grosso, Brasil.

${ }^{2}$ Mestre em Ciências Ambientais e da Saúde pela Universidade Pontifícia Universidade Católica de Goiás (PUCGO), Goiás, GO, Brasil.

${ }^{3,4}$ Mestre em Enfermagem pela Universidade Federal de Mato Grosso (UFMT), Cuiabá, Mato Grosso, Brasil.

5,6 Graduada em Enfermagem pela Universidade Federal de Mato Grosso (UFMT), Cuiabá, Mato Grosso, Brasil.
} 


\section{Introdução}

A infância consiste em um período marcado por grandes transformações, tanto no desenvolvimento físico quanto psicossocial, no qual cada criança se desenvolve de acordo com seu próprio ritmo. Esse período é dividido em quatro fases, classificadas de acordo com a faixa etária, sendo elas: lactente, infante, pré-escolar e escolar. Porém, quando ocorre um desequilíbrio na ingesta ou na absorção de nutrientes o crescimento e o desenvolvimento na infância pode se tornar inadequado, trazendo repercussões em todos os estágios do desenvolvimento, deixando-a suscetível a diversas patologias, condições e agravos, como a desnutrição e obesidade ${ }^{1-3}$.

A desnutrição, uma condição que atinge principalmente crianças abaixo de cinco anos nos países em desenvolvimento, possui origem multicausal e consiste na ingestão imprópria ou inadequada de nutrientes necessários para o metabolismo do organismo. Ela aumenta o risco de uma série de doenças, pode levar ao comprometimento crônico da nutrição, qual seja o déficit de altura/estatura para a idade, considerado um problema de saúde pública, além de consequências no desenvolvimento, colocando a criança em uma situação de vulnerabilidade $e^{4-5}$.

Por outro lado, a obesidade pode ser definida como um excesso de tecido gorduroso no organismo estando relacionado a influências e a vários fatores como genéticos, psicológicos, sedentarismo e, principalmente, pela má alimentação e erros alimentares. Na última década, observou-se um aumento significativo do número de casos de sobrepeso e obesidade em populações pediátricas, tendo ligação principalmente ao desmame precoce, a introdução de alimentos inadequados ${ }^{6}$ e a inatividade física da criança ${ }^{7}$.

Na população de baixa renda, principalmente em crianças menores de cinco anos, tem-se observado uma transição nutricional, com o aumento na prevalência de obesidade, caracterizando a mobilidade social dessa condição e a variação da morbimortalidade infantil modifica-se entre as diversas regiões e camadas da população, causando uma relação entre nutrição adequada e contexto social ${ }^{8-9}$.

Assim, o período da infância, por ser um momento marcado por um intenso crescimento e desenvolvimento, deve ser intensamente avaliado por profissionais de saúde a fim de reduzir as possíveis complicações decorrentes de alterações nutricionais comuns nessa etapa da vida. Esse acompanhamento do crescimento e desenvolvimento infantil é extremamente importante sendo uma das ações básicas da assistência integral a saúde da criança preconizada pelo Ministério da Saúde (MS), o que envolve principalmente crianças de até cinco anos de idade, considerando a alta velocidade, vulnerabilidade e intensidade do processo de crescimento e desenvolvimento nessa etapa ${ }^{8}$.

Atualmente, as creches, projetadas para ofertar condições adequadas para um desenvolvimento integral, tornaram-se uma saída para as dificuldades de implementação não somente da educação infantil, mas de proteção e acompanhamento nutricional, proporcionando, em muitos casos, acesso à alimentação adequada a crianças, independente da renda familiar ${ }^{9-11}$.

Neste sentido, esse estudo justifica-se pelo fato da avaliação antropométrica ser fundamental no diagnóstico nutricional de crianças que frequentam Unidades Municipais de Educação Infantil (UMEls), além dos fatores socioeconômicos que podem ter influência nessa condição nutricional, possibilitando a intervenção em possíveis fatores predisponentes ao desenvolvimento de doenças crônicas não transmissíveis que podem acometê-las na adolescência e/ou fase adulta.

A partir destas considerações definiu-se como questão norteadora: "Qual a relação do estado nutricional de crianças menores de 5 anos, que frequentam Unidades de Educação Infantil do município de Rondonópolis-MT, com o perfil socioeconômico de suas famílias?"

O presente estudo teve como objetivo realizar avaliação nutricional de crianças de 01 a 04 anos matriculadas em Unidades Municipais de Educação Infantil (UMEI) em dois polos da cidade de Rondonópolis/Mato Grosso e descrever a relação do perfil socioeconômico da família com o estado nutricional da criança.

\section{Metodologia}

Trata-se de um estudo exploratório descritivo com abordagem quantitativa, desenvolvido em Unidades Municipais de Educação Infantil (UMEIs), que possuem atividades de creche (para crianças até 3 anos de idade) e de pré-escola (para crianças de 4 a 5 anos de idade), localizadas no município de Rondonópolis, da região sul do estado de Mato Grosso.

O município de Rondonópolis conta com 13 UMEls distribuídas conforme a necessidade de cada região e classificadas a proximidade entre elas. Devido a grande quantidade de por Polos, constituindo assim, uma média total de 1799 crianças 
matriculadas. A cidade contém 5 polos, os quais são constituídos pelas creches de acordo com crianças atendidas no município, após um sorteio elaborado pelas pesquisadoras, foi selecionado dois polos para este estudo. O polo I é composto por 3 UMEls, e o Polo III por 2 UMEls.

Foi adotado um critério de abordagem amostral de $30 \%$ das crianças matriculadas em cada creche. Segundo Berquó ${ }^{12}$, a amostragem escolhida é classificada como Amostragem Casual Simples Estratificada com Partilha Proporcional, pois a amostragem estratificada "é aquela em que o pesquisador deseja que as subpopulações sejam representadas na amostra com a mesma proporcionalidade com que compõem a população total" Os polos selecionados e classificados como polo I e polo III têm em média 611 crianças nas UMEIs, nos quais foram selecionadas 185 crianças, perfazendo desse total, respectivamente, 96 crianças do polo I e 89 crianças do polo III, de ambos os sexos ${ }^{12}$.

Foram incluídas nessa pesquisa crianças de 12 a 59 meses de idade, que estavam matriculadas nas UMEls no ano 2013, nos referidos polos. Todos os pais ou responsáveis legais foram previamente esclarecidos pelas pesquisadoras sobre os objetivos do estudo e as técnicas as quais as crianças seriam submetidas, assinando um Termo de Consentimento Livre e Esclarecido, seguindo os padrões éticos da pesquisa com seres humanos, conforme Resolução n ${ }^{0} 466 / 12$, do Conselho Nacional de Saúde ${ }^{13}$.

Essa pesquisa foi aprovada pelo Comitê de Ética e Pesquisa (CEP) do Hospital Júlio Muller de Cuiabá, Mato Grosso, com n do Parecer Consubstanciado 247.044 do dia 26/09/2013. Ressalta-se que essa pesquisa é um recorte do projeto de pesquisa intitulado "Estado Nutricional de Crianças de 01 a 04 anos: relação entre avaliação antropométrica e perfil socioeconômico".

A coleta de dados ocorreu no período de outubro a dezembro de 2013, através de um questionário com perguntas abertas e fechadas, elaborado pelas pesquisadoras. $O$ instrumento da anotação dos dados foi um formulário contendo variáveis antropométricas (peso, altura, índice de massa corporal (IMC), perímetro cefálico e perímetro torácico), como também dados socioeconômicos das respectivas famílias (quantidade de filhos, escolaridade e ocupação dos pais, renda familiar, auxílio governamental e condições de moradia).

Para se obter os dados antropométricos, o espaço físico foi cuidadosamente preparado, a fim de não haver interferência nos dados a serem coletados. Para isso, foram utilizadas fita métrica com $150 \mathrm{~cm}$ de extensão, régua antropométrica de madeira com $100 \mathrm{~cm}$ de extensão, balança pediátrica horizontal de $16 \mathrm{~kg}$ fracionada de $100 \mathrm{~g} \mathrm{e}$ balança mecânica adulta com capacidade para $130 \mathrm{~kg}$.

A avaliação da estatura das crianças que não permaneciam em pé foi realizada em decúbito dorsal com o uso da régua antropométrica, para as crianças que já permaneciam em pé utilizou-se a régua de medidas da balança mecânica adulta. Para a mensuração do peso das crianças menores de 2 anos, foram despidas e colocadas no centro da balança, utilizando-se a balança pediátrica horizontal. Para crianças maiores de 2 ano, foram colocadas de pé, no centro da mesma, descalças e com roupas leves, empregando a balança mecânica adulta.

Para a avaliação do estado nutricional das crianças, foi utilizado o IMC [peso $(\mathrm{kg}) / a$ ltura $\left(\mathrm{m}^{2}\right)$ ], de acordo com a classificação nutricional do Caderno de Atenção Básica, Saúde da criança: Crescimento e Desenvolvimento14, e com a Caderneta de Saúde da Criança, ambos elaborados pelo Ministério da Saúde ${ }^{15}$, os quais preconizam a classificação em magreza acentuada, magreza, IMC adequado, risco de sobrepeso, sobrepeso e obesidade.

Após a avaliação, os dados foram compilados no software Excel $2010^{\circledR}$ e foi realizado a sua análise descritiva, incluindo cálculo de médias, frequências e porcentagens.

\section{Resultados}

A partir de uma população de 1799 crianças, fizeram parte da pesquisa uma amostra constituída por 185 crianças com idade entre 12 e 59 meses como apresenta a Tabela 1. Observa-se que, a maioria das crianças, $114(61,62 \%)$, matriculadas nas UMEls encontram-se nas faixas etárias de 24 a 35 meses e de 36 a 47 meses. 
Tabela 1 - Tabela de classificação de crianças por faixa etária dos Polos I e III. Rondonópolis, 2014.

\begin{tabular}{c|c|c|c|c|c}
\hline \multirow{2}{*}{$\begin{array}{c}\text { Idade } \\
\text { (meses) }\end{array}$} & \multicolumn{2}{|c|}{ Polo I } & \multicolumn{2}{c|}{ Polo III } & Total \\
\cline { 2 - 6 } & Frequência (N) & $\begin{array}{c}\text { Porcentagem } \\
(\%)\end{array}$ & Frequência (N) & $\begin{array}{c}\text { Porcentagem } \\
(\%)\end{array}$ & $(\mathrm{N})$ \\
\hline 12 a 23 meses & 19 & $19,79 \%$ & 20 & $22,48 \%$ & 39 \\
\hline 24 a 35 meses & 34 & $35,42 \%$ & 23 & $25,84 \%$ & 57 \\
\hline 36 a 47 meses & 28 & $29,17 \%$ & 29 & $32,58 \%$ & 57 \\
\hline 48 a 59 meses & 15 & $15,62 \%$ & 17 & $19,10 \%$ & 32 \\
\hline Total & 96 & $100 \%$ & 89 & $100 \%$ & 185 \\
\hline
\end{tabular}

De acordo com a Figura 1, o polo I constitui-se de 96 crianças, sendo estas, 52 do sexo masculino $(54,17 \%)$ e 44 do sexo feminino (45,83\%). No polo Ill encontram-se 89 crianças, onde 49 são do sexo masculino $(55,06 \%)$ e 40 do sexo feminino $(44,94 \%)$.

Figura 1 - Distribuição das crianças por sexo nos Polos I e III

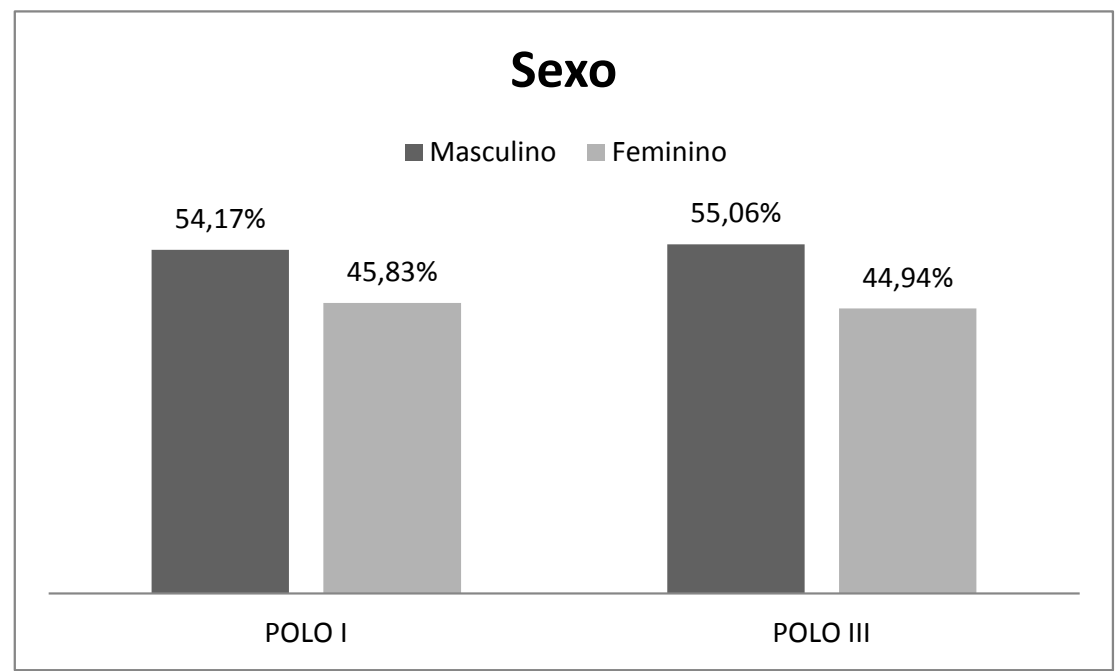

Em relação aos dados socioeconômicos das famílias entrevistadas dos polos I e III, a quantidade de filhos variou entre 1 a 8. No polo I, 28 famílias (29,17\%) possuem 2 filhos; 25 (26,04\%), 3 filhos; 18 (18,75\%), têm 1 filho; 16 (16,67\%), 4 filhos; 6 (6,25\%), têm 5 filhos; 2 (2,08\%), 7 filhos e 1 (1,04\%) têm 8 filhos. No polo III 34 famílias (38,20\%) têm 2 filhos; 23 (25,84\%), 1 filho; 19 (21,35\%), 3 filhos; 8 (8,99\%), 4 filhos; 3 famílias (3,37\%) têm 6 filhos e 2 (2,25\%) têm 5 filhos.

Dos 185 pais das crianças das UMEls entrevistados, 59 possuem o ensino médio completo $(31,90 \%)$, 38 pais têm 0 ensino fundamental incompleto (20,54\%), 26 o ensino médio incompleto (14,06\%), 20 concluíram o ensino fundamental completo (10,82\%), 13 ensino superior incompleto (7,02\%), 10 ensino superior completo $(5,40 \%), 5$ são analfabetos $(2,70 \%), 1$ tem formação latu senso $(0,54 \%)$ e 13 optaram por não responder $(7,02 \%)$.

Em relação às mães, foi possível observar que 65 delas possuem ensino médio completo $(35,16 \%), 34$ ensino fundamental incompleto (18,38\%), 33 ensino médio incompleto (17,84\%), 17 ensino fundamental completo $(9,18 \%), 12$ ensino superior incompleto (6,48\%), 9 ensino superior completo $(4,86 \%), 4$ são pós graduadas $(2,16 \%), 1$ é analfabeta $(0,54 \%)$ e 10 optaram por não responder $(5,40 \%)$.

As profissões que mais se destacaram em relação aos pais foram 21 pedreiros (11,35\%), 16 motoristas (8,64\%), e 10 vendedores 5,40\%. Entre as mães, as profissões que mais se sobressaíram foram 35 do lar (18,91\%), 15 domésticas $(8,10 \%)$ e 14 serviços gerais $(7,56 \%)$.

A renda das famílias entrevistadas variou de acordo com o polo analisado. Sendo assim, no polo I, 43 famílias possuem entre um e dois salários mínimos (44,79\%), 20 recebem entre dois e três salários mínimos (20,83\%), 13 entre três e quatro salários mínimos (13,54\%), 13 até um salário mínimo (13,54\%), 6 entre quatro e cinco salários mínimos (6,25\%), e 1 família recebe acima de cinco salários mínimos (1,05\%). No polo III, 32 famílias recebem entre um e dois salários mínimos (35,96\%), 30 entre dois e três salários mínimos (33,70\%), 11 entre três e quatro salários mínimos (12,36\%), 9 até um salário mínimo $(10,12 \%), 6$ entre quatro e cinco salários mínimos (6,74\%) e 1 família recebe acima de cinco salários mínimos (1,12\%). 
Em relação ao total de famílias analisadas do polo I, 48 delas recebem o auxílio governamental denominado Bolsa Família $(50,0 \%), 47$ famílias não recebem nenhum tipo de benefício $(48,96 \%)$ e 1 família recebe outro tipo de benefício não especificado (1,04\%); no polo III, 64 famílias não recebem nenhum tipo de benefício (71,92\%), 23 recebem auxílio governamental tipo Bolsa Família (25,84\%) e 2 famílias recebem outro tipo de benefício não especificado $(2,24 \%)$, como mostra a Figura 2.

Figura 2 - Famílias que recebem auxílio financeiro do governo conforme os Polos I e III

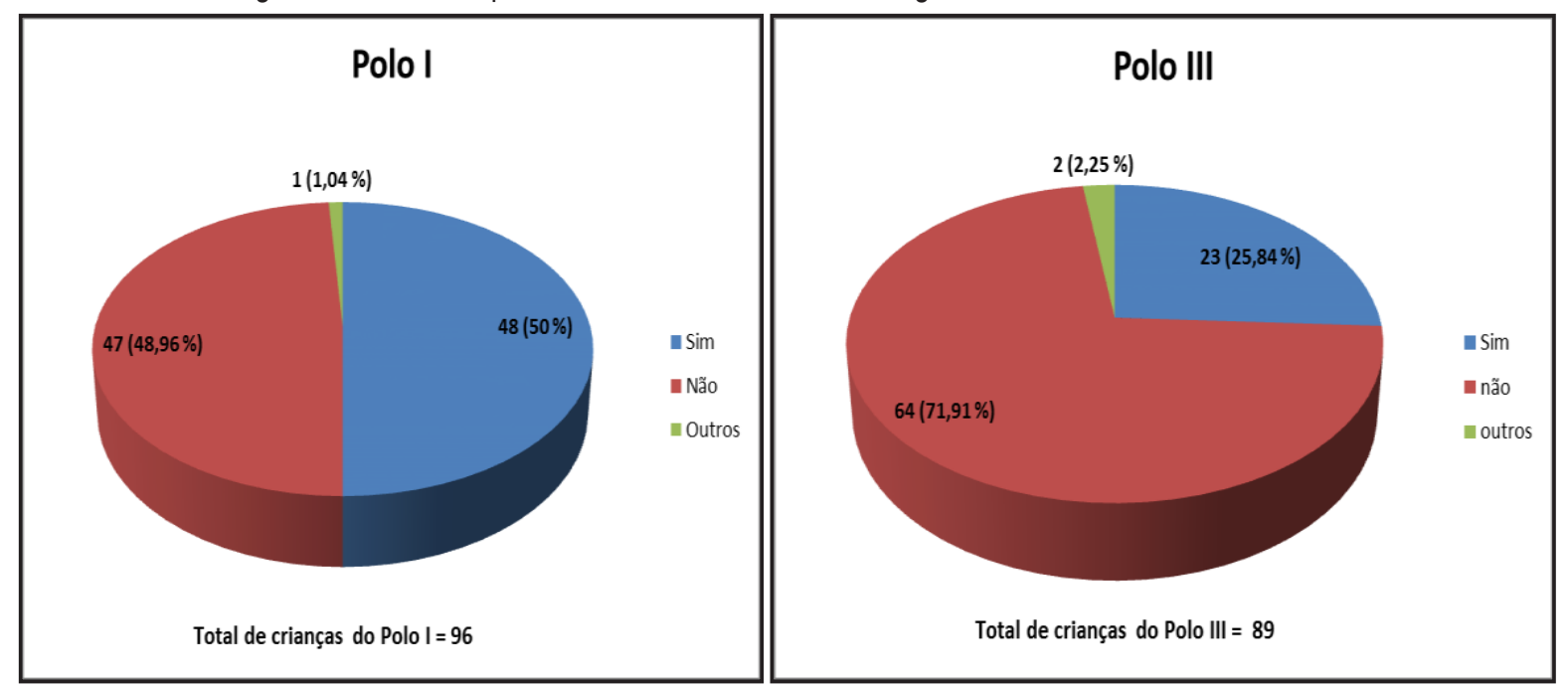

No polo I, 71 famílias possuem moradia própria (73,96\%), 17 possuem casa alugada (17,70\%) e 8 famílias moram em casa cedida (8,34\%). Em relação ao polo III, 64 famílias possuem casa própria $(71,91 \%), 17$ famílias possuem casa alugada $(8,99 \%)$ e 8 famílias moram em casa cedida (8,99\%).

A Tabela 2 apresenta os resultados da análise do estado nutricional das crianças dos Polos I e III, sendo considerado o peso e a altura para realizar o cálculo do IMC. É possível verificar que no Polo I e III a classificação nutricional predominante entre as crianças foi "IMC adequado" (85,42\% e 57,30\% respectivamente), porém a classificação que não houve ocorrência de sujeitos para o Polo I foi "Obesidade" e para o Polo III "Magreza acentuada".

Tabela 2 - Classificação nutricional das crianças dos Polos I e III de acordo com IMC. Rondonópolis, 2014.

\begin{tabular}{c|c|c|c|c}
\hline \multirow{2}{*}{$\begin{array}{c}\text { Classificação } \\
\text { nutricional }\end{array}$} & \multicolumn{2}{|c|}{ Polo I } & \multicolumn{2}{c}{ Polo III } \\
\cline { 2 - 5 } & Frequência (N) & $\begin{array}{c}\text { Porcentagem } \\
(\%)\end{array}$ & Frequência (N) & $\begin{array}{c}\text { Porcentagem } \\
(\%)\end{array}$ \\
\hline Magreza acentuada & 2 & $2,08 \%$ & 0 & $0 \%$ \\
\hline Magreza & 5 & $5,21 \%$ & 7 & $7,87 \%$ \\
\hline IMC adequado & 82 & $85,42 \%$ & 51 & $57,30 \%$ \\
\hline Risco de sobrepeso & 6 & $6,25 \%$ & 20 & $22,47 \%$ \\
\hline Sobrepeso & 1 & $1,04 \%$ & 8 & $8,99 \%$ \\
\hline Obesidade & 0 & $0 \%$ & 3 & $3,37 \%$ \\
\hline Total & 96 & $100 \%$ & 89 & $100 \%$ \\
\hline
\end{tabular}

\section{Discussão}

A partir da amostra estudada, constata-se que a faixa etária predominante das crianças matriculadas em UMEls do município de Rondonópolis é de 24 a 47 meses de vida. Nesses primeiros anos de vida, a criança possui especial sensibilidade aos estímulos oriundos do ambiente o que gera importantes influências na formação motora, física, mental e social ${ }^{16}$. 
Conforme Constituição Federal Brasileira de 1988, Dos Direitos Sociais, Artigo $7^{\circ}$, inciso XXV, os trabalhadores em geral, que visem a sua melhoria social, têm direito ao atendimento em creches e pré-escolas aos filhos e seus dependentes, de 0 a 5 anos de idade ${ }^{17}$.

Desde então, o direito à educação para as crianças na faixa etária compreendida entre 0 e 5 anos de idade vem recebendo especial atenção por parte do legislador, sendo inserido na Lei de Diretrizes e Bases - Lei no 9.394/96 que descreve, em seu artigo 29, que a "educação infantil, primeira etapa da educação básica, tem como finalidade 0 desenvolvimento integral da criança de até 5 (cinco) anos, em seus aspectos físico, psicológico, intelectual e social, complementando a ação da família e da comunidade"18.

A pesquisa também revelou que foi encontrado um índice maior de crianças do sexo masculino do que do sexo feminino em relação a quantidade de crianças avaliadas, isso pode estar relacionado ao número populacional que a cidade apresenta, onde, de acordo com dados do IBGE, a população masculina da cidade de Rondonópolis encontra-se em maior número ${ }^{19}$.

Outro fator que chamou a atenção é a quantidade de filhos nessas famílias, que variou entre um a oito, porém com maior prevalência de dois filhos por família. Esse dado pode relacionar-se ao período em que as famílias se encontram atualmente, no qual as mulheres postergam a maternidade e se introduzem no mercado de trabalho a fim de complementar a renda familiar, buscando cada vez mais uma segurança socioeconômica, e muitas se tornam chefes de família.

Um estudo realizado em creches públicas do município de Araraquara-SP demonstrou associação significativa entre o estado nutricional de pré-escolares e o número de pessoas residentes no domicílio, encontrando, ao contrário do esperado, número significativo de crianças com pré-obesidade e menor número de crianças eutróficas ${ }^{20}$.

Quanto ao nível de escolaridade das famílias entrevistadas predominou ensino médio completo, seguido de ensino fundamental incompleto, ainda, um pai possui pós graduação e, em relação às mães, esse número sobe para quatro. Se utilizarmos os dados do IBGE como parâmetro, inferimos que as famílias usuárias dessas creches apresentam o grau de escolaridade acima da população mato-grossense, que possui, em sua maioria, ensino fundamental incompleto ${ }^{19}$.

Neste estudo as profissões que mais se destacaram, em relação aos pais, foram pedreiro, motorista e vendedor. Entre as mães, as profissões que mais se sobressaíram foram do lar, doméstica e serviços gerais. Quanto a renda mensal, verificou-se que as famílias entrevistadas recebem em média de 1 a 2 salários mínimos.

$\mathrm{Na}$ Pesquisa Nacional por Amostra de Domicílios (PNAD) são encontradas diferentes ocupações na relação de trabalho entre empregado e empregador, em que o trabalhador doméstico possui grande destaque, sendo incluída nessa categoria empregada doméstica, faxineira, motorista, babá, mordomo, entre outros. Com isso, pode-se observar que as profissões mais citadas pelos entrevistados são também as mais encontradas pela PNAD/2013, que vem ganhando destaque dentre a participação dos domésticos na população ocupada ${ }^{21}$.

A hipótese de que a renda familiar influencia no estado nutricional de uma criança, no que tange 0 acesso a uma alimentação adequada, foi confirmada em estudo que demonstrou ser significativa a associação entre a renda familiar per capita e a situação de insegurança alimentar, interferindo no poder de compra das pessoas ${ }^{22}$. Assim, as condições sociais e econômicas das famílias, especialmente as características das mães das crianças, interferem no estado nutricional infantil, na ocorrência de distúrbios tanto de carências nutricionais, quanto de excesso de peso ${ }^{23}$.

Em relação ao total de famílias beneficiárias do programa bolsa família, pode-se dizer que, no polo I, área mais periférica da cidade, há maior número de famílias que recebem esse auxílio governamental (50\%), enquanto no polo III, área mais centralizada, o número de famílias beneficiárias é menor $(25,84 \%)$. Isso pode ser explicado devido ao objetivo desse programa, que é a transferência de renda para promover o alívio imediato da pobreza, as condições ao acesso a direitos sociais básicos nas áreas de educação, saúde e assistência social e o desenvolvimento das famílias, de modo que os beneficiários consigam superar a situação de vulnerabilidade ${ }^{24}$.

Sendo um dos objetivos deste programa reduzir a fome no Brasil, uma das condições para a permanência dos beneficiários é o acompanhamento do estado nutricional, para isso deve ser garantido aos mesmos acesso a serviços de qualidade com avaliação e intervenção adequadas, para que possa haver a efetivação das metas ${ }^{25}$.

É importante destacar que os programas de intervenção (como por exemplo o Programa bolsa família) devem incentivar a família a ter uma maior interação entre a criança, família e a sociedade. E que as creches devem ser não só favorecer 0 aspecto nutricional, a aprendizagem a orientação psicopedagógica, mas sim incentivar o desenvolvimento independente do nível socioeconômico da população envolvida ${ }^{16}$.

Para o acompanhamento do crescimento e desenvolvimento infantil, com o intuito de intervenções precisas e eficazes caso haja necessidade, é de fundamental importância a verificação periódica das medidas antropométricas, sendo essencial e prática para avaliação do estado nutricional da criança, antes de recorrer a avaliação sobre consumo alimentar, que é mais específica ${ }^{25-26}$. 
Neste estudo destacam-se os resultados da avaliação do estado nutricional das crianças, inferindo que a maioria delas apresenta-se dentro dos padrões de normalidade para o peso e a altura, ou seja, prevalece a ocorrência de IMC adequado. No entanto, nesta pesquisa a população infantil ainda apresenta relevante índice para risco de sobrepeso, sobrepeso e obesidade, sendo encontrados aleatoriamente esses índices nos dois polos analisados.

Observou-se que a renda familiar predominante dos Polos I e III é de 1 a 2 salários mínimos e refletiu em índices nutricionais adequados para as crianças pesquisadas. Esse resultado satisfatório pode estar relacionado com a frequência dessas crianças em UMEls, que possuem grande relevância social, pois permanecem em período integral ou parcial, sendo este um ambiente fundamental para o favorecimento do estado nutricional e do desenvolvimento neuropsicomotor ${ }^{16}$.

Atualmente, a obesidade vem ganhando destaque em estudos epidemiológicos, pois se tornou um problema de saúde pública nas últimas décadas, com índices aumentados em todo o mundo, inclusive nos países em desenvolvimento como o Brasil, onde tempos atrás os problemas de saúde estavam relacionados a desnutrição ${ }^{27}$.

Embora esses países estejam passando por uma transição nutricional, ou seja, destacando-se por seus inúmeros registros de sobrepeso e obesidade ${ }^{28-29}$, ainda é possível encontrar casos de crianças com índices de magreza acentuada, principalmente em regiões menos favorecidas, geralmente determinada pela má alimentação.

Contudo, ainda devemos considerar preocupante a desnutrição infantil, que pode ser diagnosticada a partir do retardo do crescimento da criança, comprometendo os mecanismos de imunidade, que é um fator de risco para doenças infecciosas e de mortalidade precoce. Além disso, a desnutrição pode causar danos ao sistema nervoso central que compromete o desenvolvimento psicomotor, pré-dispõe a doenças crônicas não transmissíveis, refletindo, assim, na capacidade de produção na vida adulta ${ }^{30-31}$.

Atualmente, estudos apontam tendência a diminuição da prevalência da desnutrição infantil em diversas partes do mundo, inclusive no Brasil, associada a melhorias de condições sociais, educativas e econômicas, que vêm ocorrendo em especial nas grandes metrópoles. Esse fenômeno é caracterizado como transição nutricional. Porém, a desnutrição ainda representa um desafio para diversas nações, persistindo entre crianças oriundas de áreas socialmente mais vulneráveis mesmo frente ao maior acesso aos alimentos em nível familiar ${ }^{31}$.

Alguns estudos mostram que mesmo nos países desenvolvidos ou em desenvolvimento, alguns fatores influenciam no peso corporal das pessoas: o nível econômico, o acesso a serviços, as condições de moradia, a disponibilidade de alimentos e acesso à informação 28 .

A tendência dos países subdesenvolvidos e em desenvolvimento é que a obesidade na infância mostre-se cada vez mais prevalente nas classes econômicas elevadas. Já nos países desenvolvidos a obesidade infantil ocorre mais entre indivíduos de baixa renda, devido ao aumento da acessibilidade a alimentos com elevado teor calórico, que promovem a sociedade, são mais palatáveis de baixo custo, porém com menor poder nutritivo ${ }^{28,32}$.

\section{Considerações Finais}

A avaliação nutricional pode ser apontada como referência no diagnóstico nutricional, devido seu baixo custo, fácil acesso e a aplicabilidade sendo que a partir dela, foi possível identificar dados que revelam a transição nutricional infantil. Verificou-se menor incidência de crianças com índices de magreza, e em maior prevalência a presença de crianças apresentando risco para sobrepeso, sobrepeso e obesidade, consolidando-se como o agravo nutricional mais importante, sendo associado a uma alta incidência de doenças crônicas não transmissíveis.

Foi possível observar que as condições socioeconômicas também influenciam diretamente na classificação nutricional da criança, pois quando a família possui uma renda desfavorável, possivelmente, acaba tendo dificuldades em oferecer uma alimentação rica em nutrientes variados para a criança. Em contrapartida, em busca de aumentar a renda familiar, as mães se introduzem no mercado de trabalho cada vez mais e, após longas jornadas, optam por oferecer aos filhos alimentos de fácil e rápido preparo, como os enlatados e industrializados, ricos em sódio, açúcares e carboidratos, desvalorizando assim, a necessidade nutricional para o desenvolvimento da criança saudável.

A escolaridade dos pais também parece estar diretamente relacionada com o estado nutricional da criança, pois existe uma compreensão de que um nível de instrução maior e adequado possibilita aos pais oferecerem aos seus filhos uma alimentação mais equilibrada, de acordo com as necessidades do período da infância.

Sendo assim, esse estudo pode demonstrar a importância do auxílio financeiro na vida de famílias brasileiras beneficiadas pelo Programa Bolsa Família, que cumpriu seu principal objetivo de combater a fome, promover a segurança 
alimentar e nutricional e retirar a população brasileira da margem de miséria absoluta. Além disso, foi possível perceber o bom emprego desse apoio monetário pelas famílias favorecidas na alimentação, podendo ser observada no resultado favorável do Índice de Massa Corpórea nas UMEls que mais recebiam o benefício.

Conclui-se então que a avaliação nutricional é uma ferramenta extremamente importante para acompanhar 0 desenvolvimento infantil, sendo que nesta pesquisa, a maioria das crianças possui o IMC adequado para a idade, o que pode estar relacionado ao perfil socioeconômico das famílias das crianças das UMEls deste município.

\section{Referências Bibliográficas}

1. Stefane JMJ. A enfermagem, o crescimento e desenvolvimento infantil. In: Schimtz EMR. A enfermagem em pediatria e puericultura. São Paulo: Atheneu; 2005.

2. Ribeiro OM, Sigaud CHS, Rezende MA, Veríssimo MLOR. Desenvolvimento Infantil: a criança nas diferentes etapas de sua vida. In: Fujimori E, Ohara CVS, organizadores. Enfermagem e a saúde da criança na atenção básica. Barueri: Manole; 2009.

3. Figueiredo NMAD. Ensinando a cuidar da criança. São Paulo: Yendis; 2010.

4. Jesus GM, Castelão ES, Vieira TO, Gomes DR, Vieira GO. Déficit nutricional em crianças de uma cidade de grande porte do interior da Bahia, Brasil. Rev. Ciênc. Saúde Coletiva. 2014;19(5):1581-8.

5. Souza OF, Benício MHD, Castro TG, Muniz PT, Cardoso MA. Desnutrição em crianças menores de 60 meses em dois municípios no estado do Acre: prevalência em fatores associados. Rev. Bras. Epidemiol. 2012;15(1): 211-21.

6. Vicari EC. Aleitamento materno, a introdução da alimentação complementar e sua relação com a obesidade infantil. Rev. Bras. Obesidade, Nutrição e Emagrecimento. 2013; 40 (7):72-83.

7. Paes ST, Marins JCB, Andreazzi AE. Efeitos metabólicos do exercício físico na obesidade infantil: uma visão atual. Rev. Paul Pediatr. 2015; 33 (1):122-9.

8. Alves AMA. A criança sadia. In: Figueiredo NMA de. Ensinando a cuidar da criança. São Paulo: Yendis, 2010.

9. Souza MM, Pedraza DF, Menezes TN. Estado nutricional de crianças assistidas em creches e situação de (in)segurança alimentar de suas famílias. Rev. Ciênc. Saúde Coletiva. 2012;17(12):3425-36.

10. Valente TB, Hecktheuer LHR, Brasil CCB. Condições socioeconômicas, consumo alimentar e estado nutricional de pré-escolares pertencentes a uma creche. Rev. Alim.Nutr. 2010;21(3):421-8.

11. Silva CR, Martins BAET, Oliveira VLMI, Miyasaka CK. Consumo alimentar e estado nutricional de pré-escolares de um centro de educação infantil do município de São Paulo. Rev. Alim.Nutr. 2010;21(3)407-13.

12. Berquó ES. Bioestatística. $2^{\mathrm{a}}$ ed. São Paulo: EPU; 2006.

13. Brasil. Ministério da Saúde. Conselho Nacional de Saúde. Resolução n.466 de 12 de dezembro de 2012. Dispõe sobre pesquisa em seres humanos e atualização do Decreto 196 de 1996. Brasília: 2012.

14. Brasil. Ministério da Saúde. Secretaria de Atenção à Saúde. Departamento de Atenção Básica. Saúde da criança: crescimento e desenvolvimento. Brasília: 2012.

15. Brasil. Ministério da Saúde. Caderneta de Saúde da Criança. 8.ed. Brasília: 2013.

16. Freitas SC, Costa GA, Formiga CKMR. Perfil do crescimento e desenvolvimento neuropsicomotor de crianças que frequentam creches municipais de Goiânia. Rev. Movimenta. 2011;4(1):4-18.

17. Brasil. Constituição 1988. Constituição da República Federativa do Brasil. Brasília: Senado Federal: Centro Gráfico; 1988.

18. Brasil. Lei n. 9394, de 20 de dezembro de 1996. Lei de diretrizes e bases da educação nacional. Presidência da República, Casa Civil. Brasília: 1996.

19. Instituto Brasileiro de Geografia e Estatística. Censo Demográfico 2010. [acesso 2014 Jun 28] Disponível em: http:// cidades.ibge.gov.br/xtras/temas. php?lang=\&codmun=510760\&idtema=1\&search=mato-grosso|rondonopolis|censodemografico-2010:-sinopse .

20. Foschini ALR, Campos JADB. Indicadores antropométricos do estado nutricional de pré-escolares em Araraquara, SP. Alim.e Nutr. 2010;21(3):349-55.

21. Instituto Brasileiro de Geografia e Estatística - IBGE. Pesquisa nacional por amostra de domicílios: PNAD 2013. Rio de Janeiro: IBGE; 2013.

22. Monteiro F, Schmidt ST, Costa IB, Almeida CCB, Matuda NS. Bolsa Família: insegurança alimentar e nutricional de crianças menores de cinco anos. Rev. Ciênc. Saúde Coletiva. 2014;19(5):1347-57. 
23. Moraes AAR. Apontamentos sobre a educação infantil. Centro de Apoio Oferacional da Infância e Juventude, Educação, Família e Sucessões do Ministério Público do Estado do Rio Grande do Sul. Rio Grande do Sul: Ministério Público do Estado do RS; 2012.

24. Brasil. Ministério do Desenvolvimento Social e Combate à fome. Bolsa Família. Brasília: 2013. [acesso 2014 Out 24]. Disponível em: http://www.mds.gov.br/bolsafamilia.

25. Wolf MR, Barros Filho AA. Estado nutricional dos beneficiários do Programa Bolsa Família no Brasil - uma revisão sistemática. Rev. Ciênc. Saúde Coletiva. 2014;19(5):1331-8.

26. Baratieri T, Soares LG, Botti ML, Campanini AC. Consulta de enfermagem em puericultura: um enfoque nos registros de atendimentos. Rev Enferm UFSM. 2014;4(1):206-16.

27. Enes CC, Slater B. Obesidade na adolescência e seus principais fatores determinantes. Rev. Bras. Epidemiol. 2010;13(1):163-71.

28. Silva DAS. Sobrepeso e obesidade em crianças de cinco a dez anos de idade beneficiárias do programa bolsa família no estado de Sergipe, Brasil. Rev. Paul Pediatr. 2011;29(4):529-35.

29. Pelegrini A, Silva DA, Petroski EL, Gaya AC. Sobrepeso e obesidade em escolares brasileiros de sete a nove anos: dados do projeto Esporte Brasil. Rev. Paul Pediatr. 2010;28:290-5.

30. Lima ALL, Silva ACF, Konno SC, Conde WL, Benicio MHD, Monteiro CA. Causas do declínio acelerado da desnutrição no Nordeste do Brasil (1986-1996-2006). Rev. Saúde Pública. 2010;44(1):17-27.

31. Vieira VL, Souza JMP, Cevato-Mancuso AN. Insegurança alimentar, vínculo mãe-filho e desnutrição infantil em área de alta vulnerabilidade social. Rev. Bras. Saúde Mater. Infant. 2010;10(2):199-207.

32. Silva DA, Pelegrini A, Petroski EL, Gaya AC. Comparação do crescimento de crianças e adolescentes brasileiros com curvas de referência para crescimento físico: dados do Projeto Esporte Brasil. J. Pediatr. 2010;86:115-20.

\section{Michele Salles da Silva}

Endereço para correspondência - Rua: Pinheiros, n¹13, Bairro: Coophalis, CEP: 78740-510, Cidade: Rondonópolis, MT, Brasil.

E-mail: michelesalles@yahoo.com.br

Lattes: http://lattes.cnpq.br/8248578412766240

Débora Aparecida da Silva Santos - deboraassantos@hotmail.com Jacqueline Pimenta Navarro - jacpimenta@hotmail.com

Gênesis Vivianne Soares Ferreira Cruz - geviferreira@gmail.com

Dangllie Katherine Verdi - dangllie@hotmail.com

Amanda de Oliveira Delfino - amandaeduda2001@hotmail.com

\section{Enviado em 15 de dezembro de 2014. Aceito em 20 de setembro de 2015.}


